\title{
Comparative Effects of Physical Exercise and Estradiol Therapy on Mitochondrial Density, Redox and Inflammatory Status in the Skeletal Muscle of Ovariectomized Rats
}

\section{Sara Barros Silva}

Universidade Federal dos Vales do Jequitinhonha e Mucuri (UFVJM)

Kinulpe Honorato-Sampaio

Universidade Federal dos Vales do Jequitinhonha e Mucuri (UFVJM)

Sabrina Paula Costa

Universidade Federal dos Vales do Jequitinhonha e Mucuri (UFVJM)

Talita Emanuela Domingos

Universidade Federal dos Vales do Jequitinhonha e Mucuri (UFVJM)

Timilly Mayra Martins da Cruz

Universidade Federal dos Vales do Jequitinhonha e Mucuri (UFVJM)

Cíntia Maria Rodrigues

Universidade Federal dos Vales do Jequitinhonha e Mucuri (UFVJM)

Karine Beatriz Costa

Universidade Federal dos Vales do Jequitinhonha e Mucuri (UFVJM)

Jousielle Márcia dos Santos

Universidade Federal dos Vales do Jequitinhonha e Mucuri (UFVJM)

Vanessa Kelly da Silva Lage

Universidade Federal dos Vales do Jequitinhonha e Mucuri (UFVJM)

Thais Peixoto Gaiad

Universidade Federal dos Vales do Jequitinhonha e Mucuri (UFVJM)

Ana Paula Santos

Universidade Federal dos Vales do Jequitinhonha e Mucuri (UFVJM)

Marco Fabrício Dias Peixoto

Universidade Federal dos Vales do Jequitinhonha e Mucuri (UFVJM)

Cândido Celso Coimbra

Universidade Federal de Minas Gerais (UFMG)

Adelina Martha dos Reis

Universidade Federal de Minas Gerais (UFMG)

Raphael Escorsim Szawka

Universidade Federal de Minas Gerais (UFMG) 


\section{Pedro Henrique Scheidt Figueiredo}

Universidade Federal dos Vales do Jequitinhonha e Mucuri (UFVJM)

\section{Henrique Silveira Costa}

Universidade Federal dos Vales do Jequitinhonha e Mucuri (UFVJM)

\section{Murilo Xavier Oliveira}

Universidade Federal dos Vales do Jequitinhonha e Mucuri (UFVJM)

\section{Vanessa Amaral Mendonça}

Universidade Federal dos Vales do Jequitinhonha e Mucuri (UFVJM)

Ana Cristina Rodrigues Lacerda ( $\sim$ lacerda.acr@ufvjm.edu.br)

Universidade Federal dos Vales do Jequitinhonha e Mucuri (UFVJM)

\section{Research Article}

Keywords: Ovariectomy, endurance training, oxidative stress, mitochondria density, cytokines, skeletal muscle.

Posted Date: February 2nd, 2022

DOI: https://doi.org/10.21203/rs.3.rs-1279791/v1

License: (c) (i) This work is licensed under a Creative Commons Attribution 4.0 International License. Read Full License 


\section{Abstract \\ Background}

Previous studies have highlighted the positive effects of estrogen replacement therapy during menopause. In addition, a growing body of evidence has also proven several beneficial effects of other therapeutic strategies, especially physical exercise, to counteract the deleterious effects of menopause on skeletal muscle. However, a comparison of the effects of exercise training versus hormone replacement therapy after menopause on muscle mitochondrial profile and redox/inflammatory status has not been investigated to date. The purpose of the study was to investigate the effects of endurance training versus estradiol therapy on mitochondrial density, redox status, and inflammatory biomarkers in the skeletal muscle of ovariectomized rats.

\section{Methods}

Thirty twelve weeks-old female Wistar rats were randomly assigned into three groups: Untrained ovariectomized rats (OVX-NAT); untrained ovariectomized rats with estrogen (ES) replacement (OVX-ER); and, trained ovariectomized rats (OVX-AT). After ovariectomy, The OVX-ER rats were treated with ES (subcutaneously implanted Silastic $®$ capsule containing $360 \mu \mathrm{g}$ of $17 \beta$-estradiol/mL) while the OVX-AT group performed a training protocol (50 70\% of maximal running speed on a treadmill, $60 \mathrm{~min} /$ day, five days/week for eight weeks). All animals performed a maximal treadmill test before and after the training protocol. After euthanasia, the soleus muscle was processed for histological e biochemical evaluations.

\section{Results}

The maximal oxygen consumption (VO2max), mitochondrial muscle density, total antioxidant capacity (FRAP), catalase (CAT) activity, and interleukin 10 levels improved only in the OVX-AT group, while only the OVX-ER rats presented an increase in CAT activity and a decrease in interleukin 6 levels.

\section{Conclusions}

Both endurance exercise training and estradiol therapy improve redox and inflammatory balance in the skeletal muscle of ovariectomized rats; however, only exercise increases VO2max and mitochondrial muscle density.

\section{Background}

The postmenopausal period is associated with several metabolic and musculoskeletal chronic disorders, such as osteoporosis, tendinopathies, and arthritis ${ }^{1,2}$. Because skeletal muscle plays a central role in musculoskeletal disorders, maintaining muscle integrity after menopause is essential to prevent future 
disabilities ${ }^{1}$. The mechanisms through which ovarian hormone deficiency negatively affects the muscle during the postmenopausal period are multifactorial, involving a pro-inflammatory profile and mitochondrial dysfunction ${ }^{1,2}$. Studies using ovariectomized (OVX) rats, the most common experimental menopause model, have reported that these rats present a reduction in mitochondrial biogenesis markers and respiratory capacity and an increase in skeletal muscle oxidative stress ${ }^{3-5}$. In addition, ovariectomy alters the intramuscular levels of several cytokines. For example, ovariectomy enhances tumor necrosis factor-alpha (TNF-a) expression, a pro-inflammatory cytokine associated with reduced muscle function 6,7 , reduces interleukin 10 (IL-10) levels, a predominant anti-inflammatory cytokine, and increases interleukin 6 (IL-6) levels, a modulatory cytokine associated with energy metabolism, inflammatory control and muscle repair. ${ }^{8,9}$. Additionally, chronic stimulation of pro-inflammatory cytokines, e.g., TNF-a, impairs mitochondrial function and biogenesis, promoting a vicious pro-inflammatory cycle ${ }^{10,11}$.

Many studies have highlighted the positive effects of estrogen replacement therapy during menopause $e^{4,5}$. Moreover, a growing body of evidence has also proven several beneficial effects of other therapeutic strategies, especially physical exercise, to counteract the deleterious effects of menopause on skeletal and cardiac muscle including a recent work of our team which evidenced beneficial effects in cardiovascular function aspects in isolated cardiac muscle ${ }^{5,6,7}$. However, a comparison of the effects of exercise training versus hormone replacement therapy after menopause on muscle mitochondrial profile and redox/inflammatory status has not been investigated to date.

Given that endurance exercise training stimulates mitochondrial biogenesis and improves redox and inflammatory status in the skeletal muscle under normal and physiological conditions ${ }^{13-15}$, we aimed to investigate the effects of exercise training omy versus hormone replacement therapy with ES on muscle mitochondrial density and redox/inflammatory status in ovariectomized rats.

\section{Methods}

\section{Animals}

12-week-old females Wistar rats ( $n=30$, body mass $=216 \pm 2.54 \mathrm{~g}$ ) were provided by the animal facility of the Universidade Federal de Minas Gerais, Brazil. The age of the rats used in this study was determined in accordance with previous studies ${ }^{4,16,17}$ and based on the premise that with 12 weeks of age, Wistar female rats are sexually mature ${ }^{17}$.

The animals were kept in a temperature-controlled room $(22 \circ \mathrm{C})$, with a $12 \mathrm{~h}$ dark: light cycle, and received standard chow and water ad libitum. All rats were treated similarly in terms of daily manipulation. All surgical procedures and protocols used were approved by Animal Use Ethics Committee of the Universidade Federal dos Vales do Jequitinhonha e Mucuri (protocol $n^{\circ} 015 / 2019$ ) and conducted in accordance with National Institute of Health $(\mathrm{NIH})$ Guide for the Care and Use of Laboratory Animals.

\section{Experimental design and sampling}


The rats were randomly assigned into three groups: 1 ) Ovariectomized not aerobically trained rats (OVXNAT, $n=10), 2$ ) Ovariectomized rats with estrogen replacement (OVX-ER, $n=10)$ and 3), Ovariectomized aerobically trained rats $(\mathrm{OVX}-\mathrm{AT}, \mathrm{n}=10)$. All animals were submitted to ovariectomy and received a subcutaneously implanted Silastic ${ }^{\circledR}$ capsule containing 17ß-estradiol (OVX-ER group) or vehicle (OVXNAT and OVX-AT groups). Two weeks after ovariectomy, OVX-AT rats were submitted to an endurance training protocol for eight weeks. The OVX-NAT and OVX-ER groups were equally handled and placed near the treadmill during the training sessions of the TR-OVX group to match the same environmental exposure conditions. All animals were submitted to exercise familiarization and to the maximal effort treadmill test before and after the endurance training protocol.

The rats were euthanized by decapitation. Both right and left soleus muscles were harvest, washed in icecold PBS (0.15M, pH 7.34), frozen in liquid nitrogen, and stored at $-80^{\circ} \mathrm{C}$. The left soleus muscles were processed for oxidative stress evaluation and the right ones for inflammatory analyses. In addition, posterior mid-belly fragments of the right soleus from three animals per group were dissected and chemically fixed for mitochondrial density assessment by transmission electron microscopy.

\section{Ovariectomy}

The animals were anesthetized (Ketamine $80 \mathrm{mg} / \mathrm{kg}+$ Xylazine $12 \mathrm{mg} / \mathrm{kg}$ ), both lateral abdominal walls were trichotomized and an incision was made. The ovaries were located, the oviduct was sectioned to remove the ovaries and the incisions were stitched ${ }^{3,4}$. The animals received one dose of antibiotics (Pentabiotic, $24.000 \mathrm{UI} / \mathrm{Kg}$ ) immediately after surgery, and two doses of analgesic (Flunixin meglumine, $2.5 \mathrm{mg} / \mathrm{kg}$ ), immediately and $24 \mathrm{~h}$ post-surgery. Animals had two weeks to recover from surgery before the maximal aerobic capacity test. All rats recovered successfully.

\section{Estrogen replacement}

Immediately after ovariectomy, all animals received a subcutaneously implanted Silastic $₫$ capsule containing $360 \mu \mathrm{g}$ of $17 \beta$-estradiol/mL in corn oil or vehicle (corn oil). The Silastic $\circledast$ capsules were made of 20-mm segments of Silastic ${ }^{\circledR}$ tubing (inner/outer diameter: 1.02/2.16 mm). An incision was made in the rat dorsal region $(10 \mathrm{~mm})$ to implant the Silastic ${ }^{\circledR}$ capsule using forceps ${ }^{16}$. The incision was subsequently stitched. Silastic ${ }^{\circledR}$ capsules were re-implanted after five weeks in order to maintain concentrations within the physiological range $\mathrm{e}^{16,18}$. The efficacy of this hormone replacement protocol was confirmed by previous studies $4,16,19,20$.

\section{Maximal aerobic exercise test}

All animals were adapted to the treadmill (0.3 km/h, $10 \mathrm{~min} /$ day, 5 days) (Panlab, Havard Apparatus, Spain $)^{21,22}$. This procedure is intended to familiarize the animals at running on the treadmill. In this study, all rats were familiarized successfully.

The maximal effort exercise test consisted of $0.18 \mathrm{~km} / \mathrm{h}$ increments every 3 minutes until the rat could no longer keep pace 21,22 . The purpose was to evaluate the maximal aerobic capacity and determine exercise 
training intensity. Maximal oxygen consumption during the maximal exercise test (VO2max), which represents the energetic cost, was performed by an indirect calorimeter (Panlab, Harvard Apparatus, Spain) coupled to the treadmill (airflow $=1.0 \mathrm{~L} / \mathrm{min}$ ). VO2max was measured continuously by a computerized system (Metabolism, Panlab, Harvard Apparatus, Spain) ${ }^{21}$.

Mechanical efficiency (ME) was calculated by the formula: $M E=\left(\right.$ workload / energetic cost) $\times 100^{23}$. Workload (W; kgm) was calculated as $\mathrm{W}=$ body weight $(\mathrm{kg}) \times$ TTF $\times$ treadmill speed $(\mathrm{m} . \mathrm{min}-1) \times$ sine $\theta$ (treadmill inclination), where TTF is time to fatigue (min).

\section{Endurance exercise training}

The OVX-AT group performed exercise on a motor treadmill (Insight ${ }^{\circledR}$, SP, Ribeirão Preto, Brazil) at lowmoderate intensity ( $\sim 50-70 \%$ maximal running speed) $60 \mathrm{~min}$. day, five days/week for eight weeks (total of 40 sessions), with a gradual increase in speed from 0.7 to $1.2 \mathrm{~km} / \mathrm{h}$ weekly ${ }^{21,23}$.

\section{Transmission electron microscopy}

Fragments of the soleus muscle were fixed in Karnovsky's solution (2.5\% glutaraldehyde and $2 \%$ paraformaldehyde) in $0.1 \mathrm{M}$ cacodylate buffer $\mathrm{pH} 7.4$ overnight at $4^{\circ} \mathrm{C}$. Then, samples were post-fixed in a mixture of $2 \%(\mathrm{w} / \mathrm{v})$ osmium tetroxide and $1.5 \%(\mathrm{w} / \mathrm{v})$ potassium ferrocyanide for a minimum of 2 hours to enhance the contrast of organelles. Thereafter, samples were washed in distilled water and kept in $2 \%$ uranyl acetate (en bloc staining) overnight, serially dehydrated in graded ethanol baths, and embedded in Epon 812. Finally, 50nm ultrathin sections were stained with Reynolds lead citrate. Transmission electron microscopy (TEM) was performed using a FEI Tecnai G2-12 Spirit at $80 \mathrm{kV}$. The images were acquired in a SIS-MegaView 3 CCD camera with 1376 x 1070 pixels. Twenty-four electron micrographs per animal were taken at a $\times 11.000$ magnification. Images were randomly selected from central parts of muscle fibers and were analyzed with ImageJ. Volume densities $(\mathrm{Vv})$ of mitochondria were determined with the classic point counting method using a 252-point-grid (500 x $500 \mathrm{~nm}$ grid) projected onto each image ${ }^{24,25}$.

\section{Redox status and antioxidant enzyme activities}

The Bradford method using bovine serum albumin was used as a standard to determine the sample's protein levels ${ }^{26}$. The reaction of the thiobarbituric acid with malondialdehyde was used to determine lipid peroxidation by thiobarbituric acid reactive substances (TBARS) levels ${ }^{27}$. The ferric reducing ability of plasma (FRAP), i.e., the reduction of ferric-tripyridyltriazine [Fe(III)-TPTZ] complex to ferroustripyridyltriazine [Fe(II)-TPTZ] was used to determine the total antioxidant capacity ${ }^{28}$. The quantification of superoxide dismutase activity (SOD) was based on the inhibition of the reaction between $02^{--}$and pyrogallol ${ }^{29}$. Catalase activity (CAT) was determined by measuring the decrease in $\mathrm{H}_{2} \mathrm{O}_{2}$ absorbance at $240 \mathrm{~nm}^{30}$.

\section{Inflammatory biomarkers}


Soleus muscle samples were defrosted gradually from -80 to $4^{\circ} \mathrm{C}$. Thereafter, they were homogenized in extraction solution ( $1 \mathrm{ml} /$ muscle $100 \mathrm{~g})$ containing PBS 01x (125mL), NaCl $(2.925 \mathrm{~g}), \mathrm{BSA}(0.625 \mathrm{~g})$, EDTA (46,5mg), PMSF (2.125mg), benzethonium chloride (5,6mg), Tween $20(62.5 \mu \mathrm{L})$, aprotinin $(2.5 \mu)$. The homogenate was then centrifugated at $10.000 \mathrm{xg}$ for $10 \mathrm{~min}$ at $4^{\circ} \mathrm{C}$. The supernatant was separated and used for analyses of IL-6, IL-10, and TNF-a according to the manufacturer's instructions by ELISA kits (DuoSet, R\&D Systems, United States).

\section{Statistical analyses}

Data are reported as mean \pm standard error (S.E.M.). Maximal exercise test comparisons were performed by ANOVA (two-way) followed by Dunnet post-hoc test. ANOVA one-way was used for mitochondrial density, redox status, antioxidant enzymes, and inflammatory biomarkers analyses. Confidence interval (Cl) and effect size (ES) for each significant analysis are also shown. The correlation between variables was evaluated using the Pearson coefficient. The significance level for all tests was set at $5 \%$. Statistical analyses were performed with GraphPad Prism 5.0 and Gpower 3.1.9.2.

\section{Results}

Figure 1 presents the body mass and the uterus/body mass ratio. Final body mass did not differ between groups after 10 weeks of ovariectomy (OVX-NAT: $290.50 \pm 6.38 \mathrm{~g}$; OVX-E2: $287.0 \pm 3.59 \mathrm{~g}$; OVX-AT: $293.00 \pm 3.88 \mathrm{~g}$, Fig. 1A). However, estradiol replacement increased the uterus/ body mass ratio (OVX-ER: $0.00070 \pm 0.00003 \mathrm{~g}$; OVX-NAT: $0.00030 \pm 0.00002 \mathrm{~g}$; and OVX-AT: $0.00039 \pm 0.00002 \mathrm{~g}$, Figure 1B).

Table 1 shows the results from the maximal aerobic capacity test. Despite the similar V02max, mechanical efficiency, final distance and time, and average speed before the training protocol, only the exercised group did not reduce the V02max and improved the mechanical efficiency, final distance and time, and average speed after the training protocol (Table 1).

Table 1. Exercise capacity. 


\begin{tabular}{|c|c|c|c|c|c|c|c|c|c|}
\hline Measurement & $\begin{array}{l}\text { OVX-NAT } \\
(\mathbf{n}=7)\end{array}$ & $\begin{array}{l}\text { OVX-ER } \\
(n=7)\end{array}$ & $\begin{array}{l}\text { OVX-AT } \\
(\mathbf{n}=7)\end{array}$ & $\begin{array}{l}\text { CI } \\
\text { (NAT ys ER) }\end{array}$ & $\begin{array}{l}\text { CI } \\
(\text { NAT ys AT) }\end{array}$ & ES & $\mathrm{p}^{\mathrm{l}}$ & $\mathrm{p}^{2}$ & $\mathrm{P}^{3}$ \\
\hline \multicolumn{10}{|l|}{$\begin{array}{l}\text { VO2 } \max \\
\left(\mathrm{mL} \cdot \mathrm{kg}^{-1} \cdot \mathrm{min}^{-1}\right)\end{array}$} \\
\hline Initial & $30.17 \pm 0.51$ & $30.78 \pm 0.51$ & $29.81=0.76$ & -2.53 to 3.00 & -3.76 to 1.78 & \multirow{2}{*}{1.73} & \multirow[b]{2}{*}{$<0.001$} & \multirow{2}{*}{$<0.001$} & \multirow[b]{2}{*}{0.055} \\
\hline Final & $24.29 \pm 0.62$ & $24.18 \pm 0.49$ & $29.98 \pm 1.12^{\circ}$ & -3.41 to 2.12 & 2.32 to 7.87 & & & & \\
\hline \multicolumn{10}{|l|}{$\begin{array}{l}\text { Mechanical } \\
\text { efficiency } \\
(\%)\end{array}$} \\
\hline Initial & $19.45=0.90$ & $19.01 \pm 1.02$ & $21.70=1.42$ & -0.05 to 0.06 & -0.02 to 0.08 & 0.95 & $<0.001$ & $<0.001$ & $<0.001$ \\
\hline \multicolumn{10}{|l|}{$\begin{array}{l}\text { Distance } \\
\text { (m) }\end{array}$} \\
\hline initial & $239.10 \pm 21.45$ & $251.20 \pm 24.66$ & $263.30=17.70$ & -90.97 to 121.70 & -84.10 to 128.60 & \multirow{2}{*}{1.56} & \multirow{2}{*}{$<0.001$} & \multirow{2}{*}{0.079} & \multirow{2}{*}{$<0.001$} \\
\hline Final & $136.80 \pm 18.44$ & $147.00 \pm 23.03$ & $530.40=72.85 *$ & -112.80 to 99.85 & 262.60 to 475.30 & & & & \\
\hline \multicolumn{10}{|l|}{$\begin{array}{l}\text { Time } \\
\text { (s) }\end{array}$} \\
\hline Initial & $1215.00 \pm 58.69$ & $1246.00 \pm 66.50$ & $1836.00 \pm 46.80$ & -195.90 to 268.70 & -170.50 to 294.10 & \multirow{2}{*}{1.49} & \multirow{2}{*}{$<0.001$} & \multirow{2}{*}{0.635} & \multirow{2}{*}{$<0.001$} \\
\hline Final & $888.60=70.04$ & $920.10 \pm 82.74$ & $1816.75 \pm 136.0^{*}$ & -247.80 to 216.80 & 643.40 to 1108.00 & & & & \\
\hline \multicolumn{10}{|l|}{$\begin{array}{l}\text { Speed } \\
(\mathrm{km} / \mathrm{h})\end{array}$} \\
\hline Initial & $1.31 \pm 0.04$ & $1.35=0.06$ & $1.35 \pm 0.03$ & -0.20 to $0.27^{n}$ & -0.20 to 0.27 & \multirow{2}{*}{1.41} & \multirow{2}{*}{$<0.001$} & \multirow{2}{*}{0.640} & \multirow{2}{*}{$<0.001$} \\
\hline Final & $1.08 \pm 0.06$ & $1.06 \pm 0.06$ & $1.94 \pm 0.09$ & -0.25 to $0.22^{\prime \prime}$ & 0.62 to 1.10 & & & & \\
\hline
\end{tabular}

Data reported as mean \pm S.E.M. 95\% confidence interval $(\mathrm{Cl})$. Untrained ovariectomized rats (OVX-NAT), untrained ovariectomized rats with estrogen replacement (OVX-ER), and trained ovariectomized rats (OVX-AT). $\mathrm{N}=10$ per group. Effect size (ES). $\mathrm{p} 1$ interaction, $\mathrm{p} 2$ time, $\mathrm{p} 3$ treatment. ${ }^{*} \mathrm{p}<0.05$ (OVX-AT vs OVX-NAT). Two-way ANOVA. Data reported as mean \pm S.E.M. 95\% confidence interval (Cl). Effect size (ES). p1 interaction, p2 time, p3 treatment. *p <0.05 (OVX-AT vs OVX-NAT).

Figure 2 highlights the results of muscle mitochondrial density. Of note, the TR-OVX had a higher muscle mitochondrial density ( 20\%) than the two other groups (TR-OVX: 19.48 $\pm 0.57 \%$; OVX-NAT: $15.59 \pm$ 0.47\%; OVX-ER: $16.50 \pm 0.59 \%$, Fig 2A-D).

Figure 3 shows the results of muscle redox status. The TBARS levels did not differ among the groups (OVX-NAT: $0.37 \pm 0.04$; OVX-ER: $0.35 \pm 0.01$; OVX-AT: $0.37 \pm 0.02 \mathrm{mmolMDA} / \mathrm{mg}$ protein, Fig. $3 \mathrm{~A}$ ). Only the exercised group improved the total antioxidant capacity (FRAP) levels (OVX-NAT: 436.10 \pm 25.06 ; OVX-ER: $433.20 \pm 23.94$; OVX-AT: $563.60 \pm 10.80 \mathrm{mmolFeSO} / \mathrm{L} / \mathrm{mg}$ protein, Fig. $3 \mathrm{~B}$ ) and catalase activity (OVXNAT: $0.67 \pm 0.03$; OVX-ER: $0.80 \pm 0.026$; OVX-AT: $0.96 \pm 0.04 \mathrm{nmol} / \mathrm{mg}$ protein, Fig. $3 \mathrm{C}$ ), while only the group treated with ES replacement improved the SOD activity (OVX-NAT: $1.28 \pm 0.01$; OVX-ER: $1.40 \pm 0.03$; OVX-AT: $1.33 \pm 0.00 \mathrm{USOD} / \mathrm{mg}$ protein, Fig. 3D).

Figure 4 presents the results of inflammatory biomarkers. TNF-a levels did not differ among the groups (OVX-NAT: $283.4 \pm 3.343$; OVX-ER: $284.4 \pm 6.652$; OVX-AT: $271.5 \pm 4.173$ pg/mg, Fig. 4A). However, while the IL-6 levels reduced ( 44\%) only in the OVX-ER group (OVX-NAT: $378.9 \pm 8.875$; OVX-ER: $262.3 \pm 13.13$; OVX-AT: $398.5 \pm 27.53 \mathrm{pg} / \mathrm{mg}$, Fig. $4 \mathrm{~B}$ ), the IL-10 levels increased ( $61 \%)$ only in the OVX-TA rats (OVXNAT: $232.3 \pm 5.249$; OVX-ER: $216.0 \pm 14.18$; OVX-AT: $375.9 \pm 15.97$ pg/mg, Fig. 4C).

\section{Discussion}


The present study was designed to compare the effects of endurance exercise training versus ES replacement therapy on mitochondrial density, redox status and inflammatory biomarkers in the skeletal muscle of ovariectomized rats. The most important finding is that both therapies exert beneficial effects, but exercise therapy was superior to hormone therapy. Whereas exercise training improved VO2max, skeletal muscle mitochondrial density, SOD activity, FRAP and IL-10 levels, ES therapy only increased muscle CAT activity and reduced IL-6 levels in ovariectomized rats.

Studies demonstrated that ovariectomy reduces rats' performance during maximal exercise tests 22,31 . This physical performance deterioration rats may be a consequence of the decrease in the skeletal muscle mitochondrial content and function induced by ovariectomy 4,5 . In our study, endurance exercise training effectively increased aerobic capacity and mitochondrial density, important physiological indicators of muscle mitochondrial function improvement ${ }^{32}$. On the other hand, ES replacement therapy did not alter these parameters.

To our knowledge, this is the first study highlighting the effects of endurance training versus ES replacement therapy on the skeletal muscle mitochondrial density from OVX rats using transmission electron microscopy, the "gold standard" to measure mitochondrial content. This assay exhibits more accuracy in measuring mitochondrial content than other measures, such as transcriptions factors levels, once changing these markers does not necessarily induce changes in mitochondrial content.

It is noteworthy that ovariectomy may increase mitochondrial ROS production ${ }^{3-5}$, favoring a pro-oxidant and inflammatory status leading to progressive mitochondrial dysfunction and cell death ${ }^{10,11}$.

Our results indicate that both estradiol replacement therapy and endurance exercise training may protect muscle from oxidative stress in different ways. While estradiol replacement therapy increased SOD activity, endurance exercise training increased CAT acvtivity in OVX skeletal muscle. However, only endurance exercise training promoted a significant increase in total antioxidant capacity, as showed by the increase in FRAP levels.

Our data also revealed that only ES therapy reduced IL-6 levels in the OVX skeletal muscle. The complex physiological role of IL-6 has been focus of discussion in several studies. While some studies suggest IL6 as an immune-modulatory cytokine inducing low-grade inflammation in some chronic diseases and a detrimental intramuscular modulatory factor in specific conditions such as cachexia, other studies suggest IL- 6 as a key factor inducing positive effects on muscle metabolism and myogenesis ${ }^{9}$. Despite these controversial effects, we believe that reduced levels of IL-6 in OVX skeletal muscle induced by estradiol replacement therapy are beneficial and contribute to an anti-inflammatory profile during menopause; however, a complete characterization of the modulatory role of intramuscular II-6 during ES replacement therapy in OVX rats deserve future investigations.

Although IL-6 was unchanged by endurance exercise training, OVX-AT group presented improved levels of IL-10, an essentially anti-inflammatory myokine that downregulates pro-inflammatory signalings and 
protects the muscle against oxidative damage $\mathrm{e}^{10,11,33}$. Moreover, our data showed that IL-10 levels showed a moderate positive correlation with FRAP levels (R squared $0.69, p<0.001$ ) while IL-6 showed no significant correlation with FRAP (R squared 0.40, $p<0.001$ ). Additionally, we also observed a moderate positive correlation between FRAP and V02max (squared $0.66, p<0.001$ ), and a strong positive correlation between IL-10 and V02max (squared 0.82, p < 0.001), reinforcing the contribution of endurance exercise training to the total antioxidant capacity and anti-inflammatory status.

The probable mechanisms underlying the improvement in skeletal muscle mitochondrial density, redox balance and anti-inflammatory profile induced by endurance exercise training in OVX rats must be further elucidated. As perspective, studies should investigate the long-term effects of endurance exercise training on skeletal muscle aspects in postmenopausal.

\section{Conclusion}

Overall, endurance exercise training was more effective in improving the skeletal muscle mitochondrial content and the redox and inflammatory status in the skeletal muscle of OVX rats compared to ES replacement therapy. Thus, endurance exercise training may be an alternative therapy to hormonal replacement therapy for the treatment of musculoskeletal disabilities during menopause.

\section{Declarations}

Ethics approval

All surgical procedures and protocols used were approved by Animal Use Ethics Committee of the Universidade Federal dos Vales do Jequitinhonha e Mucuri (protocol $n^{\circ} 015 / 2019$ ) and conducted in accordance with National Institute of Health $(\mathrm{NIH})$ Guide for the Care and Use of Laboratory Animals. Of note, we confirm that our work is reported as described by the ARRIVE guidelines.

\section{Consent for publication}

The researchers of this study confirm that they have given due consideration to protecting the intellectual property associated with this work and that there are no impediments to publication, including the timing of publication, with respect to intellectual property. In so doing, we confirm that we have followed the regulations of our institutions concerning intellectual property.

Availability of data and materials

The datasets used and/or analysed during the current study are available from the corresponding author on reasonable request.

Competing interests 
The authors declare that they have no known competing financial interests or personal relationships that could have appeared to influence the work reported in this paper.

The authors declare no financial interests/personal relationships that may be considered as potential competing interests.

\section{Funding}

There are currently no Funding Sources in the list.

\section{Authors' contributions}

- SBS: Formal analysis, Data Curation, Methodology, Writing- Review \& Editing - Original Draft.

- KHS: Conceptualization, Data Curation, Writing- Review \& Editing - Original Draft.

- SPC: Writing - Review \& Editing - Original Draft.

- TED: Writing- Review \& Editing - Original Draft.

- TMMC: Writing - Review \& Editing - Original Draft.

- CMR: Writing- Review \& Editing - Original Draft.

- KBC: Methodology,Writing- Review \& Editing - Original Draft.

- JMS: Methodology, Writing- Review \& Editing - Original Draft.

- VKSL: Writing- Review \& Editing - Original Draft.

- TPG: Writing - Review \& Editing - Original Draft.

- APS: Writing - Review \& Editing - Original Draft.

- MFDP: Writing- Review \& Editing - Original Draft.

- CCC: Writing - Review \& Editing - Original Draft.

- AMR: Writing- Review \& Editing - Original Draft.

- RES: Writing- Review \& Editing - Original Draft.

- PHSF: Writing- Review \& Editing - Original Draft.

- HSC: Writing- Review \& Editing - Original Draft.

- MXO: Writing- Review \& Editing - Original Draft. 
- VAM:Data Curation, Writing- Review \& Editing - Original Draft.

- ACRS: Conceptualization, Data Curation, Writing- Review \& Editing - Original Draft.

\section{Acknowledgements}

Part of this research derives from the Master's Thesis of Sara Barros Silva. We would like to thank the Federal University of the Jequitinhonha and Mucuri Valleys (Universidade Federal dos Vales do Jequitinhonha e Mucuri) for institutional support. We also thank the National Council for Scientific and Technological Development (CNPq), the Research Support Foundation for the state of Minas Gerais (FAPEMIG), and the Coordination for the Improvement of Higher Education Personnel (CAPES - finance code 001). Besides, the assistance of the Brazilian Society of Physiology is gratefully acknowledged.

\section{References}

1. K.H. Collins, W. Herzog, G.Z. MacDonald, et al. Obesity, metabolic syndrome, and musculoskeletal disease: Common inflammatory pathways suggest a central role for loss of muscle integrity. Front. Physiol. 9 (2018). https://doi.org/10.3389/fphys.2018.00112.

2. F. Lizcano, G. Guzmán, Estrogen deficiency and the origin of obesity during menopause. Biomed Res. Int. 2014 (2014). https://doi.org/10.1155/2014/757461.

3. W. Sutham, J. Sripetchwandee, W. Minta et al. Ovariectomy and obesity have equal impact in causing mitochondrial dysfunction and impaired skeletal muscle contraction in rats. Menopause. 25 (2018) 1448-1458. https://doi.org/10.1097/GME.0000000000001149.

4. J.P.A. Cavalcanti-de-Albuquerque, I.C. Salvador, E.L. Martins, et al. Role of estrogen on skeletal muscle mitochondrial function in ovariectomized rats: a time course study in different fiber types. $J$. Appl. Physiol. 116 (2014) 779-789. https://doi.org/10.1152/japplphysiol.00121.2013.

5. G. Capllonch-Amer, M. Sbert-Roig, B.M. Galmés-Pascual, et al. Estradiol stimulates mitochondrial biogenesis and adiponectin expression in skeletal muscle. J. Endocrinol. 221 (2014) 391-403. https://doi.org/10.1530/JOE-14-0008.

6. S. Dagdeviren, H.B. Kandilci, B. Uysal, N.D. Zeybek, P. Korkusuz, B. Gümüsel, F. Korkusuz, Tumor necrosis factor-alpha antagonist administration recovers skeletal muscle dysfunction in ovariectomized rats. J. Orthop. Res. 29 (2011) 275-280. https://doi.org/10.1002/jor.21226.

7. S. P. Costa, T. E. Domingues, C. M. Rodrigues, S. B. Silva, C. O. Dinis e Magalhães, L. V.Costa-Pereira, M. F. D. Peixoto, S. F. Da Fonseca, K. H. Ssampaio, V. A. Mendonça, A. C. R. Lacerda, Does endurance training prior to ovariectomy protect against myocardial contractility dysfunction in rats? Experimental Gerontology, (2021) v. 155, n. September, p. 111556.

https://doi.org/10.1016/j.exger.2021.111556

8. J.S. Kim, J. Jeon, J.J. An, H.K. Yi, Interval running training improves age-related skeletal muscle wasting and bone loss: Experiments with ovariectomized rats. Exp. Physiol. 104 (2019) 691-703. https://doi.org/10.1113/EP087458. 
9. P. Muñoz-Cánoves, C. Scheele, B.K. Pedersen, A.L. Serrano, Interleukin-6 myokine signaling in skeletal muscle: A double-edged sword?. FEBS J. 280 (2013) 4131-4148. https://doi.org/10.1111/febs.12338.

10. A.D. Cherry, C.A. Piantadosi, Regulation of Mitochondrial Biogenesis and Its Intersection with Inflammatory Responses. Antioxidants Redox Signal. 22 (2015) 965-976. https://doi.org/10.1089/ars.2014.6200.

11. A. Valerio, A. Cardile, V. Cozzi, R. Bracale,et al. TNF-a downregulates eNOS expression and mitochondrial biogenesis in fat and muscle of obese rodents. J. Clin. Invest. 116 (2006) 2791-2798. https://doi.org/10.1172/JCl28570.

12. P. Stute, I. Ceausu, H. Depypere, I. Lambrinoudaki, et al. A model of care for healthy menopause and ageing: EMAS position statement. Maturitas. 92 (2016) 1-6. https://doi.org/10.1016/j.maturitas.2016.06.018.

13. C. Lundby, R.A. Jacobs, Adaptations of skeletal muscle mitochondria to exercise training. Exp. Physiol. 101 (2016) 17-22. https://doi.org/10.1113/EP085319.

14. B.K. Pedersen, Muscles and their myokines, J. Exp. Biol. 214 (2011) 337-346. https://doi.org/10.1242/jeb.048074.

15. B. Egan, J.R. Zierath, Exercise metabolism and the molecular regulation of skeletal muscle adaptation. Cell Metab. 17 (2013) 162-184. https://doi.org/10.1016/j.cmet.2012.12.012.

16. M.R. Barbosa, G.E. Shiguemoto, L.M. Tomaz, et al. Resistance Training and Ovariectomy: Antagonic Effects in Mitochondrial Biogenesis Markers in Rat Skeletal Muscle. Int. J. Sports Med. 37 (2016) 841-848. https://doi.org/10.1055/s-0042-107247.

17. H. Quinteiro, M. Buzin, F.F. Conti, D.D.S. Dias, et al. Aerobic exercise training promotes additional cardiac benefits better than resistance exercise training in postmenopausal rats with diabetes. Menopause. 22 (2015) 534-541. https://doi.org/10.1097/GME.0000000000000344.

18. J.O. Ström, E. Theodorsson, A. Theodorsson, Order of magnitude differences between methods for maintaining physiological $17 \beta$-oestradiol concentrations in ovariectomized rats. Scand. J. Clin. Lab. Invest. 68 (2008) 814-822. https://doi.org/10.1080/00365510802409703.

19. N. Yousefzadeh, K. Kashfi, S. Jeddi, A. Ghasemi, E. Physiology, B. Sciences, B. Education, E. Physiology, A. Street, D. Blvd, Review article: ovariectomised rat model of osteoporosis. EXCLI J. 19 (2020) 89-107.

20. A. Pighon, J. Gutkowska, M. Jankowski, R. Rabasa-Lhoret, J.M. Lavoie, Exercise training in ovariectomized rats stimulates estrogenic-like effects on expression of genes involved in lipid accumulation and subclinical inflammation in liver. Metabolism. 60 (2011) 629-639. https://doi.org/10.1016/j.metabol.2010.06.012.

21. C.G. Leandro, A.C. Levada, S.M. Hibara, R. Manhães-De-Castro, C.B. De-Castro, R. Curi, T.C. PithonCuri, A program of moderate physical training for Wistar rats based on maximal oxygen consumption. J. Strength Cond. Res. 21 (2007) 751-756. https://doi.org/10.1519/00124278200708000-00016. 
22. C. Mostarda, V. Salemi, J. Freire Machi, P. Lázara Cruz, K. De Angelis, O. Albuquerque de Moraes, M. Barbosa da Silva, S. Freitas, M.-C. Irigoyen, D. da Silva Dias, M. Morris, Impact of aging on cardiac function in a female rat model of menopause: role of autonomic control, inflammation, and oxidative stress. Clin. Interv. Aging. (2016) 341. https://doi.org/10.2147/cia.s88441.

23. G.A. Brooks, C.M Donovan and T.P White. Estimation of anaerobic energy production and efficiency in rats during exercise. J.Appl.Physiol. 56,520-525 https://doi.org/10.1109/T-ED.1984.21594

24. K. Honorato-Sampaio, K. Barbosa de Queiroz, D. Andrade Leal, L. Kappes-Becker, J.V. Rossoni Júnior, R. Guerra-Sá, A.B.G. Pinto, E.A. Evangelista, Physical activity prevents alterations in mitochondrial ultrastructure and glucometabolic parameters in a high-sugar diet model. PLoS One. 12 (2017) e0172103. https://doi.org/10.1371/journal.pone.0172103.

25. N.T. Broskey, J. Daraspe, B.M. Humbel, F. Amati, Skeletal muscle mitochondrial and lipid droplet content assessed with standardized grid sizes for stereology. J. Appl. Physiol. 115 (2013) 765-770. https://doi.org/10.1152/japplphysiol.00063.2013.

26. M.M. Bradford, A Rapid and Sensitive Method for the Quantitation of Microgram Quantities of Protein Utilizing the Principle of Protein-Dye Binding. Anal. Biochem. 72 (1976) 248-254. https://doi.org/10.1016/j.cj.2017.04.003.

27. H. Ohkawa, N. Ohishi, K. Yagi, Assay for lipid peroxides in animal tissues by thiobarbituric acid reaction. Anal. Biochem. 95 (1979) 351-358. https://doi.org/10.1016/0003-2697(79)90738-3.

28. J.J. Benzie, Iris F. F.; Strain, The Ferric Reducing Ability of Plasma (FRAP) as a Measure of "'Antioxidant Power"': The FRAP Assay. Anal. Biochem. 239 (1996) 70-76. https://doi.org/10.1006/abio.1996.0292.

29. S.L. Marklund, Superoxide dismutase isoenzymes in tissues and plasma from New Zealand black mice, nude mice and normal BALB/c mice. Mutat. Res. - Fundam. Mol. Mech. Mutagen. 148 (1985) 129-134. https://doi.org/10.1016/0027-5107(85)90216-7.

30. H. Aebi, [13] Catalase in Vitro. Methods Enzymol. 105 (1984) 121-126. https://doi.org/10.1016/S0076-6879(84)05016-3.

31. F.F. Conti, I.C. Sanches, N. Bernardes, G.L. Shimojo, D.M.T. Figueroa, R.B. Wichi, K. De Angelis, Influence of sexual hormones on oxygen consumption in rats. Rev. Bras. Med. Do Esporte. 20 (2014) 421-423. https://doi.org/10.1590/1517-86922014200601665.

32. N.T. Broskey, A. Boss, E.J. Fares, C. Greggio, G. Gremion, L. Schlüter, D. Hans, R. Kreis, C. Boesch, F. Amati, Exercise efficiency relates with mitochondrial content and function in older adults. Physiol. Rep. 3 (2015) 1-9. https://doi.org/10.14814/phy2.12418.

33. S. Dagdeviren, D.Y. Jung, R.H. Friedline, H.L. Noh, et al. IL-10 prevents aging-associated inflammation and insulin resistance in skeletal muscle. FASEB J. 31 (2017) 701-710. https://doi.org/10.1096/fj.201600832R.

\section{Figures}


(A)

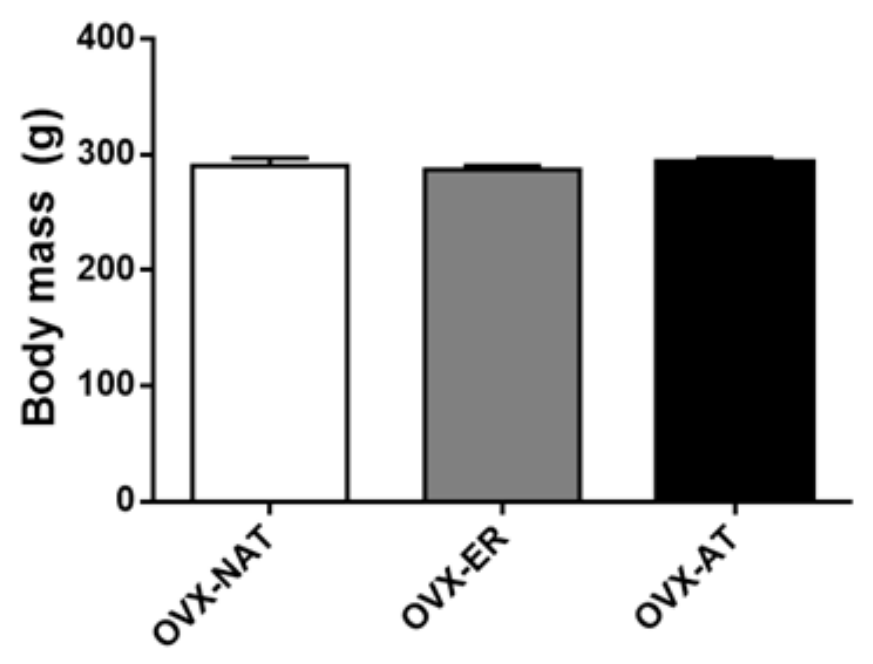

(B)

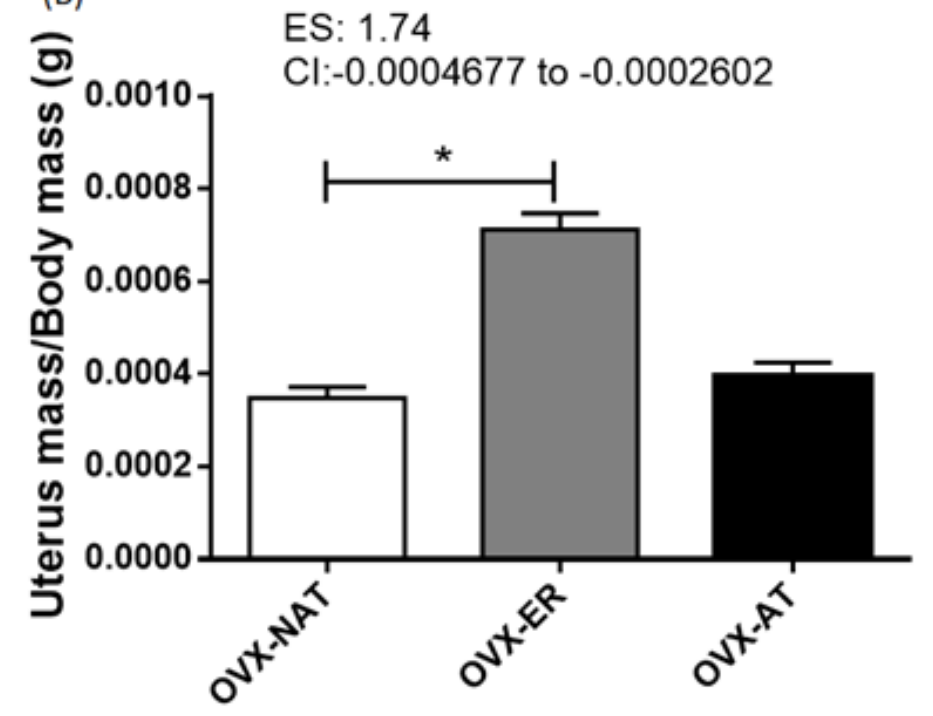

\section{Figure 1}

(A) Body mass and (B) uterus mass/body mass of untrained ovariectomized rats (OVX-NAT), untrained ovariectomized rats with estrogen replacement (OVX-ER), and trained ovariectomized rats (OVX-AT). N = 10 per group. Data are reported as mean \pm S.E.M. $95 \%$ confidence interval $(\mathrm{Cl})$. Effect size $(\mathrm{ES}){ }^{*} \mathrm{p}<0.05$ (OVX-AT vs OVX-ER). One-way ANOVA.

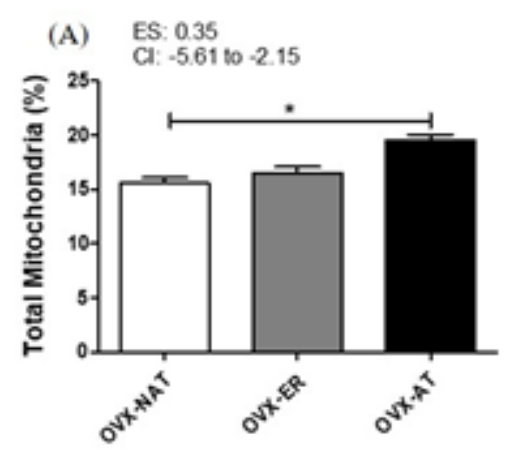

(B) OVX-NAT

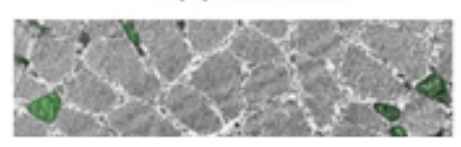

(C) OVX-ER

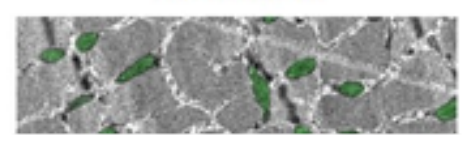

(D) OVX-AT

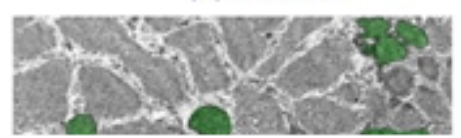

Figure 2

Mitochondrial muscle density. A). Quantification of total mitochondria muscle density in mid-belly fragments of the right soleus of untrained ovariectomized rats (OVX-NAT), untrained ovariectomized rats 
with estrogen replacement (OVX-ER), and trained ovariectomized rats (OVX-AT). $N=72$ fields from three animals per group. B, C, D) Transmission electron micrographs (TEM) of transverse sections of muscle fibers (ultra-structural view), in which mitochondria is highligthted in green. Data are reported as mean \pm S.E.M. 95\% confidence interval (Cl). Effect size (ES) * $p<0.01$. One-way ANOVA.

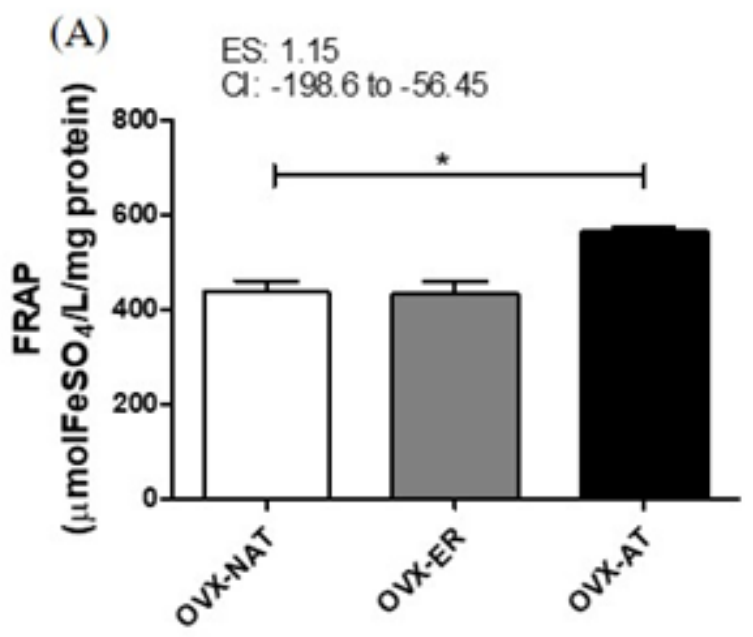

(B)

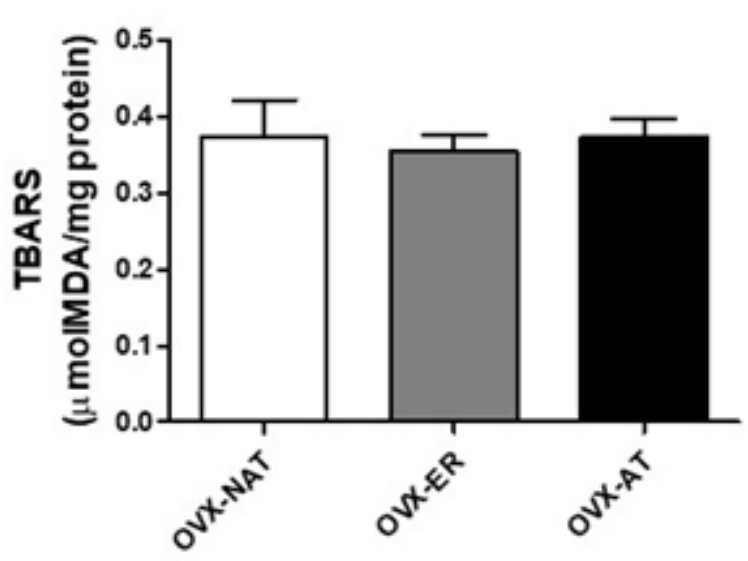

(C)

ES: 0.85

(D)

C: -0.2034 to -0.03318
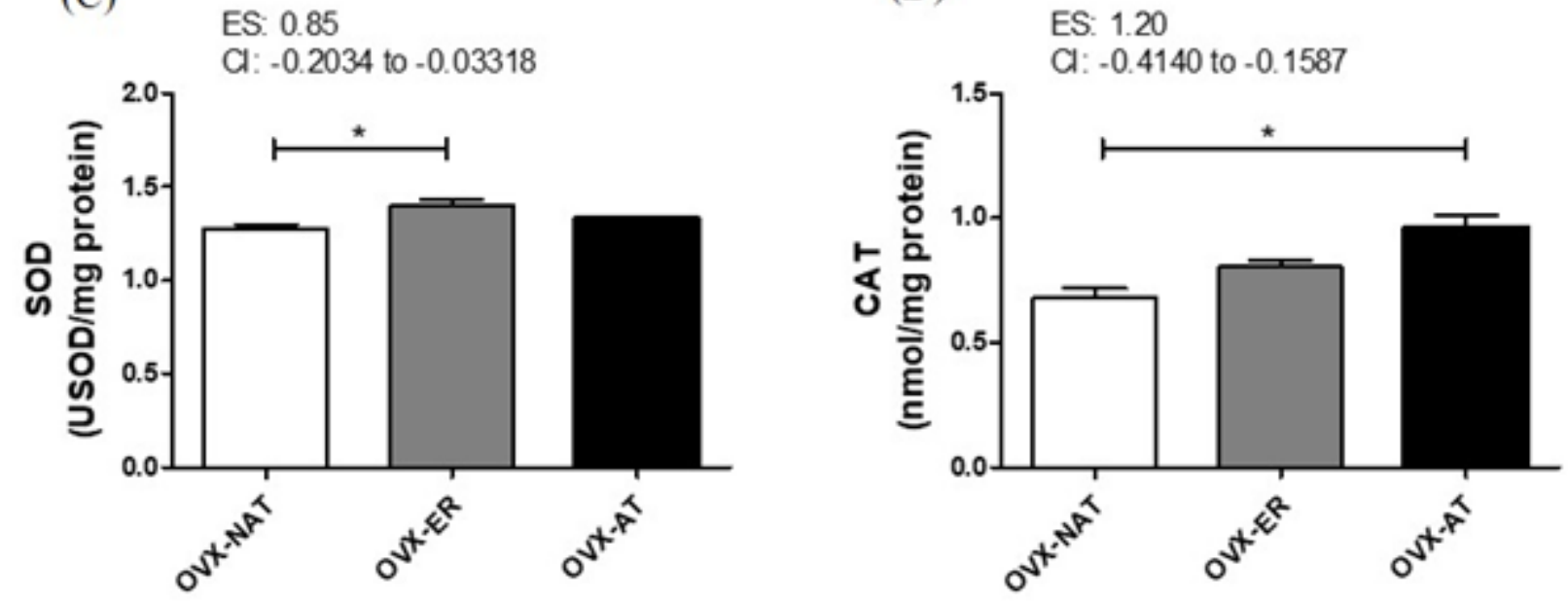

Figure 3

Redox status and anti-oxidative enzymes assays in the left soleus muscle of untrained ovariectomized rats (OVX-NAT), untrained ovariectomized rats with estrogen replacement (OVX-ER), and trained ovariectomized rats (OVX-AT).A). Ferric reducing ability of plasma(FRAP). B). Thiobarbituric acid reactive substances assay (TBARS). C). Superoxide dismutase activity (SOD). D). Catalase activity (CAT). $N=10$ per group. Data are reported as mean \pm S.E.M. 95\% confidence interval $(\mathrm{Cl})$. Effect size $(\mathrm{ES}){ }^{*} \mathrm{p}<0.01$. One-way ANOVA. 
(A)

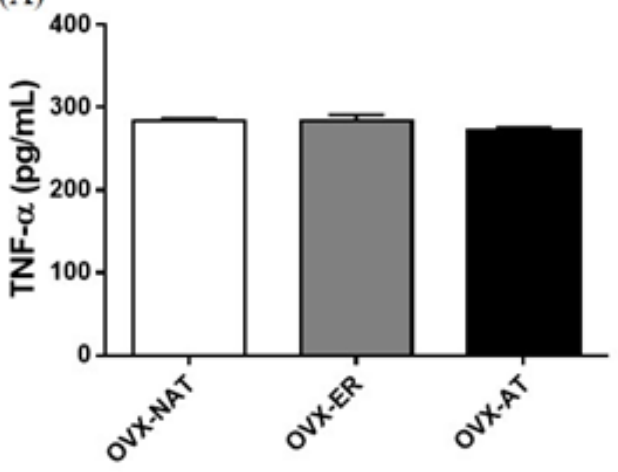

ES: 1.37

(B) $\quad$ Cl: 54.46 to 178.9

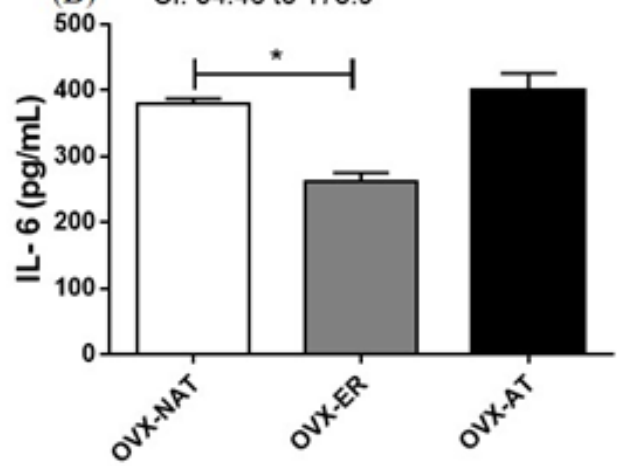

ES: 2.30

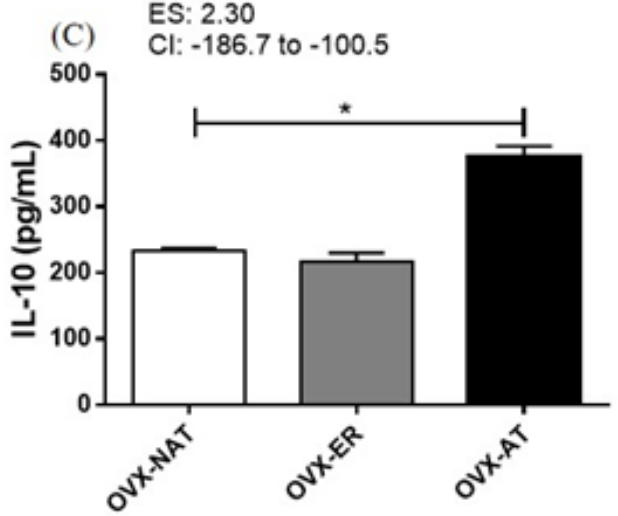

\section{Figure 4}

Inflammatory biomarkers assays in the right soleus muscle of ovariectomized rats (OVX-NAT), untrained ovariectomized rats with estrogen replacement (OVX-ER), and trained ovariectomized rats (OVX-AT). A). Tumor necrosis factor-alpha (TNF-a).B). Interleukin 6 (IL-6). C). Interleukin 10 (IL-10). N = 10 per group. Data are reported as mean \pm S.E.M. $95 \%$ confidence interval $(\mathrm{Cl})$. Effect size $(\mathrm{ES}) * p<0.01$. One-way ANOVA. 